\title{
The Role of Endoscopic Ultrasound-Guided Ki67 in the Management of Non-Functioning Pancreatic Neuroendocrine Tumors
}

\author{
YongYan Cui ', Lauren G. Khanna ${ }^{2}$, Anjali Saqi ${ }^{3}$, John P. Crapanzano ${ }^{3}$, James M. Mitchell ${ }^{3}$, Amrita Sethi ${ }^{4}$, Tamas A. Gonda ${ }^{4}$, \\ Michael D. Kluger ${ }^{5}$, Beth A. Schrope ${ }^{5}$, John Allendorf ${ }^{6}$, John A. Chabot ${ }^{5}$ and John M. Poneros ${ }^{4}$ \\ ${ }^{1}$ Department of Medicine, New York Presbyterian Columbia University Irving Medical Center, New York, NY, ${ }^{2}$ Division of Gastroenterology \\ and Hepatology, Department of Medicine, New York University, New York, NY, ${ }^{3}$ Department of Pathology and Cell Biology, New York \\ Presbyterian Columbia University Irving Medical Center, New York, NY, ${ }^{4}$ Division of Digestive and Liver Diseases, Department of Medicine, \\ New York Presbyterian Columbia University Irving Medical Center, New York, NY, ${ }^{5}$ Department of Surgery, New York Presbyterian Columbia \\ University Irving Medical Center, New York, NY, ${ }^{6}$ Department of Surgery, New York University Winthrop Hospital, Mineola, NY, USA
}

Background/Aims: The management of small, incidentally discovered nonfunctioning pancreatic neuroendocrine tumors (NF-PNETs) has been a matter of debate. Endoscopic ultrasound with fine-needle aspiration (EUS-FNA) is a tool used to identify and risk-stratify PNETs. This study investigates the concordance rate of Ki67 grading between EUS-FNA and surgical pathology specimens in NFPNETs and whether certain NF-PNET characteristics are associated with disease recurrence and disease-related death.

Methods: We retrospectively reviewed the clinical history, imaging, endoscopic findings, and pathology records of 37 cases of NFPNETs that underwent pre-operative EUS-FNA and surgical resection at a single academic medical center.

Results: There was 73\% concordance between Ki67 obtained from EUS-FNA cytology and surgical pathology specimens; concordance was the highest for low- and high-grade NF-PNETs. High-grade Ki67 NF-PNETs based on cytology $(p=0.028)$ and histology $(p=0.028)$ were associated with disease recurrence and disease-related death. Additionally, tumors with high-grade mitotic rate $(p=0.005)$, tumor size $>22.5 \mathrm{~mm}(p=0.104)$, and lymphovascular invasion $(p=0.103)$ were more likely to have poor prognosis.

Conclusions: NF-PNETs with high-grade Ki67 on EUS-FNA have poor prognosis despite surgical resection. NF-PNETs with intermediate-grade Ki67 on EUS-FNA should be strongly considered for surgical resection. NF-PNETs with low-grade Ki67 on EUSFNA can be monitored without surgical intervention, up to tumor size $20 \mathrm{~mm}$. Clin Endosc 2020;53:213-220

Key Words: Concordance; Ki67; Neuroendocrine; Nonfunctioning

\section{INTRODUCTION}

Malignant pancreatic neuroendocrine tumors (PNETs) are clinically rare and heterogeneous neoplasms that represent $<3 \%$ of all pancreatic malignancies. ${ }^{1,2}$ PNETs are clinically

Received: March 19, 2019 Accepted: April 6, 2019

Correspondence: YongYan Cui

Department of Medicine, New York Presbyterian Columbia University Irving Medical Center, 321 E. 25th St. Apt 5D, New York, NY 10010, USA

Tel: +1-312-420-4006, Fax: +1-312-586-7981, E-mail: yycui90@gmail.com ORCID: https://orcid.org/0000-0002-3883-2721

(c) This is an Open Access article distributed under the terms of the Creative Commons Attribution Non-Commercial License (http://creativecommons.org/ licenses/by-nc/3.0) which permits unrestricted non-commercial use, distribution, and reproduction in any medium, provided the original work is properly cited. divided into two categories: functioning and nonfunctioning. While functioning PNETs secrete hormones that cause symptoms, nonfunctioning PNETs (NF-PNETs) are generally asymptomatic until they grow large enough to cause mass effect or until they metastasize. The incidence of PNETs is $<1 / 100,000$ cases annually, although autopsy studies have found prevalence rates between 0.8 to $10 \% .^{1-5}$ In the last decade, increased use of cross-sectional imaging has led to a steady rise in diagnosis of NF-PNETs due to incidental detection. $^{6-8}$

Surgery has been the standard management for PNETs. For functioning PNETs that are symptomatic, surgical resection is curative. However, for NF-PNETs that are small, asymptomatic, and vary in growth rate and malignant potential, it is 
unclear when surgery has to be indicated. Although some case series have indicated that surgical resection is correlated with increased overall survival of NF-PNET patients, ${ }^{9-11}$ more recent studies have demonstrated that small NF-PNETs (variably defined as $<3.0 \mathrm{~cm}$ ) have low risk of progression and metastasis and may not benefit from surgery. ${ }^{12-16}$ Additionally, these studies report that these small tumors $(<2.0 \mathrm{~cm})$ are more likely to be incidentally discovered and benign. Thus, although the National Comprehensive Cancer Network recommends surgical resection for most patients with localized disease, it states that observation is an option for small incidentally discovered NF-PNETs. ${ }^{17}$ Similarly, the 2016 European Neuroendocrine Tumor Society (ENETS) Consensus Guidelines urged physicians to weigh the risk-benefit ratio of surgery for asymptomatic tumors less than $2.0 \mathrm{~cm} .^{18}$

Ki67 is a marker of cell proliferation and has been used in PNET grading, with higher values indicating more aggressive behavior. Numerous studies investigating NF-PNETs have found tumor diameter, Ki67 proliferative index $>2 \%$, and/or presence of lymph node disease to be predictive of malignant potential and worse outcomes. ${ }^{1,57,9,19-25}$ Endoscopic ultrasound with fine-needle aspiration (EUS-FNA) is a validated method used to identify, measure, sample, and risk-stratify all PNETs according to these prognostic factors. ${ }^{23,26,27}$ Prior studies have evaluated the concordance between Ki67 indices obtained from EUS-FNA cytology and from surgical pathology specimens in all (functioning and nonfunctioning) PNETs. ${ }^{828-34}$ These studies have found Ki67 concordance rates ranging between $69.2 \%$ and $92 \%$. Ki67 discordance between cytology and histology is seen primarily in intermediate- and highgrade tumors that have higher tumor heterogeneity and larger tumor size. ${ }^{8,27,34}$ Thus far, only Larghi et al. has investigated Ki67 concordance between cytology and histology specifically in NF-PNETs. ${ }^{29}$ Further investigation of these factors may be key to assessing malignant potential and determining management of small NF-PNETs.

This is the largest single-center study investigating the concordance rate of Ki67 grading between EUS-FNA cytology and surgical pathology specimens in NF-PNETs. This study also investigates whether NF-PNET characteristics such as tumor size, mitotic rate, lymph node involvement, and lymphovascular invasion during surgical resection can lead to poor clinical outcomes such as disease recurrence and disease-related death.

\section{MATERIALS AND METHODS}

This study design was approved by the Human Subjects Institutional Review Board of the New York Presbyterian-Co- lumbia University Irving Medical Center. All cases of EUSFNA involving pancreatic lesions performed at our hospital between January 2005 and December 2015 were retrospectively reviewed. Patients with NF-PNETs who underwent both pre-operative EUS-FNA and surgical resection were identified. Pathology records were reviewed and, in cases without Ki67 immunohistochemistry, Ki-67 immunostain was performed. Paucicellular cytology specimens (i.e., $<200$ cells) and cases without cell blocks for Ki67 immunohistochemistry were excluded. Additionally, in an attempt to keep the sample uniform, PNET patients with multiple endocrine neoplasia type I syndrome or mixed cellular features (i.e., neuroendocrine with adenocarcinoma and/or acinar cell carcinoma) were excluded. A total of 37 cases fulfilled the inclusion criteria.

EUS-FNA was performed by, or under the supervision of, three advanced endoscopists. The needle size, needle type, and number of passes were determined at the discretion of the endoscopists.

Immunohistological staining for Ki67 was performed on the cytology and surgical pathology specimens using clone 30-9 on Benchmark Ultra platform using Ultraview diaminobenzidine tetrahydrochloride detection kit from Ventana. Staining was evaluated by three cytopathologists and/or pathologists experienced in gastrointestinal surgical pathology. Samples were graded according to the 2017 World Health Organization (WHO) criteria. Ki67 indices of EUS-FNA and surgical resection specimens were each classified as low$(<3 \%)$, intermediate- $(3 \%-20 \%)$, or high-grade $(>20 \%)$ based on manual counting. Mitotic rates of surgical resection specimens were classified as low- $(<2$ per 10 high power field [HPF]), intermediate- (2-20 per $10 \mathrm{HPF})$, or high-grade ( $>20$ per 10 HPF).

\section{Statistical analysis}

All patient cases were included in statistical analysis comparing concordance rates of (1) Ki67 grading between EUSFNA cytology and surgical pathology specimens and (2) Ki67 grading and mitotic rate grading of surgical pathology specimens.

All patients, except one patient with synchronous metastasis during diagnosis, were included in the Kaplan-Meier estimator for disease recurrence. Fisher's exact test was used for univariate analysis to determine the risk factors associated with disease-related death and disease recurrence. One patient had a NF-PNET resection with disease recurrence at seven years after surgical resection. This patient's second tumor data was excluded from receiver operating characteristic (ROC) curve analysis and Fisher's exact test. $P$-values less than 0.05 were regarded as statistically significant. Given our sample size, odds ratios were calculated with a two-by-two table instead of 
logistic regression.

All data were analyzed with SPSS 24.0 for Windows.

\section{RESULTS}

\section{Patients and EUS procedures}

From 2005 to 2015, 36 patients (62\% female; median age, 62 years; range, $38-83$ years) with NF-PNETs underwent both EUS-FNA and surgical resection of their tumors. One patient was included twice as she developed recurrence seven years after her initial surgical resection; she underwent both EUSFNA and surgical resection during recurrence.

Tumor size ranged from 7 to $170 \mathrm{~mm}$ (median, $25 \mathrm{~mm}$; mean, $40 \mathrm{~mm})$. Thirteen tumors (35.1\%) were located in the head, 23 (62.2\%) were located in the body and tail, and 1 was found throughout the entire pancreas (size, $65 \mathrm{~mm}$ ). At the time of resection, positive lymph nodes were found in $11(29.7 \%)$ cases, lymphovascular invasion was present in 17 (45.9\%), and synchronous metastasis was present in 1 (2.7\%) case (Table 1).

Needle sizes used for EUS-FNA sampling included 19 G,

Table 1. Non-Functioning Pancreatic Neuroendocrine Tumor Characteristics $(n=37)$

\begin{tabular}{lrc}
\hline Characteristics & $n$ & $\%$ \\
\hline $\begin{array}{l}\text { Ki67 grading based on surgical resection } \\
\text { specimen }\end{array}$ & 24 & \\
$\quad$ Low grade (<3\%) & 8 & 21.6 \\
Intermediate grade (3\%-20\%) & 5 & 13.5 \\
High grade (20\%) & & \\
Mitotic rate based on surgical resection spec- & & \\
imen & 27 & 73.0 \\
Low grade (<2 per 10 HPF) & 4 & 10.8 \\
Intermediate (2-20 per 10 HPF) & 6 & 16.2 \\
High & & \\
Location & 13 & 35.1 \\
Head & 23 & 62.2 \\
Body and tail & 1 & 2.7 \\
Entire pancreas & & \\
Size & & \\
$\leq 16.5$ mm & 30 & 81.1 \\
$>16.5$ mm & 7 & 18.9 \\
Surgery findings & & \\
Positive lymph nodes & 17 & 29.7 \\
Lymphovascular invasion & & \\
\hline
\end{tabular}

HPF, high power field.

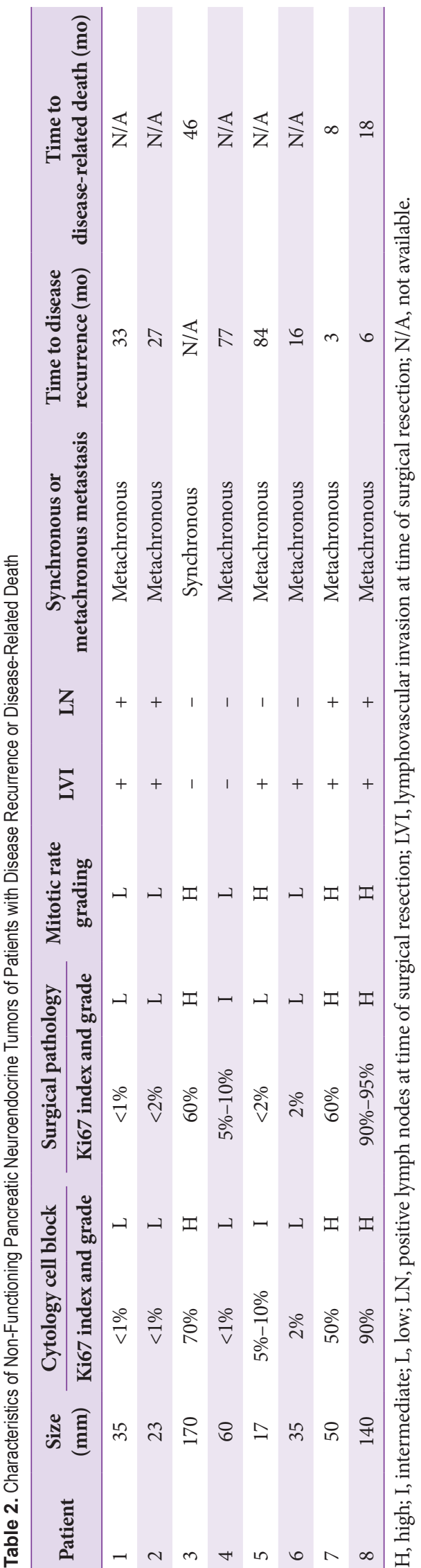


$22 \mathrm{G}$, and $25 \mathrm{G}$. In 16 cases, only $25 \mathrm{G}$ needles were used; in 8 cases, only $22 \mathrm{G}$ needles were used; in the remaining cases, a combination of needle sizes was used. Number of passes ranged from 1 to 9 passes, with a mean and median of 5 passes. Time from the EUS-FNA to surgical resection ranged from 3 days to 115 days, with an average of 31.2 days.

\section{Clinical outcome}

Five patients were lost to follow-up within three months following resection. For those who remained in follow-up, average follow-up was 49.9 months (range, 4.6 to 134.1 months) and average disease-free survival was 53.9 months.

One patient had high-grade Ki67 and synchronous metastasis during diagnosis and died eventually. Seven patients developed disease recurrence following resection (range, 3 to

Table 3. Concordance of Grading between Surgical Pathology Ki67 versus EUS-FNA Ki67 versus Surgical Mitotic Rate

\begin{tabular}{llccc}
\hline & & \multicolumn{3}{c}{ Surgical pathology Ki67 } \\
\cline { 3 - 5 } & & Low & Intermediate & High \\
\hline EUS-FNA Ki67 & Low & $20^{\text {a) }}$ & 5 & 0 \\
& Intermediate & 4 & $3^{\text {a) }}$ & 1 \\
& High & 0 & 0 & $4^{\text {a) }}$ \\
\multirow{2}{*}{$\begin{array}{c}\text { Surgical } \\
\text { mitotic rate }\end{array}$} & Low & $22^{\text {a) }}$ & 5 & 0 \\
& Intermediate & 1 & $3^{\text {a) }}$ & 0 \\
Total & High & 1 & 0 & $5^{\text {a) }}$ \\
\hline
\end{tabular}

EUS-FNA, endoscopic ultrasound with fine-needle aspiration.

${ }^{a}$ Indicates concordance with surgical pathology Ki67 grading.
84 months). Six of these patients had lymphovascular invasion and four patients concomitantly had positive lymph nodes during initial resection. In two cases with high-grade Ki67, time to disease recurrence was $\leq 6$ months and disease-related death was the eventual clinical outcome. This is compared to four low-grade cases and one intermediate-grade Ki67 case that had disease recurrence between 16 and 84 months (Table 2).

\section{Concordance of Ki67 grading between EUS-FNA cytology and surgical resection specimen histology}

Twenty-seven out of 37 cases (73.0\%) had concordant Ki67 grades between cytology and histology (Table 3). Concordance was the highest in (20/24) low-grade tumors and (4/5) high-grade tumors, with positive predictive values of $80.0 \%$ and $100 \%$ respectively. Only 3 of 8 cases of intermediate tumors were concordant, with a positive predictive value of $37.5 \%$.

In the ten discordant cases, four tumors graded as intermediate by EUS-FNA were downgraded to low after surgical resection; five tumors graded as low by EUS-FNA were upgraded to intermediate after surgical resection; and one tumor that was graded as intermediate by EUS-FNA was upgraded to high after surgical resection.

In all cases that were upgraded, tumor size was equal to or greater than $25 \mathrm{~mm}$. In cases that were downgraded, tumor size ranged from $10 \mathrm{~mm}$ to $140 \mathrm{~mm}$. Number of passes and location did not differ significantly in discordant cases. In cases that were later upgraded, number of passes ranged from three to nine. In cases that were later downgraded, number of passes ranged from two to eight (Table 4). Time between EUS-FNA and surgical resection did not differ significantly

Table 4. Characteristics of Discordant Ki67 Cases

\begin{tabular}{|c|c|c|c|c|c|c|}
\hline Patient case & $\begin{array}{c}\text { EUS-FNA Ki67 } \\
\text { grade }\end{array}$ & $\begin{array}{l}\text { Surgical pathology } \\
\text { Ki67 grade }\end{array}$ & $\begin{array}{c}\text { Tumor size } \\
(\mathbf{m m})\end{array}$ & Tumor location & Number of passes & Needle size(s) \\
\hline \multicolumn{7}{|l|}{ Upgraded } \\
\hline 1 & $\mathrm{~L}$ & I & 60 & Head & 3 & $25 \mathrm{G}$ \\
\hline 2 & $\mathrm{~L}$ & I & 55 & Tail & 3 & $22 \mathrm{G}$ \\
\hline 3 & I & $\mathrm{H}$ & 25 & Body & 5 & $22 \mathrm{G}$ \\
\hline 4 & $\mathrm{~L}$ & I & 27 & Head & 9 & $25 \mathrm{G}$ \\
\hline 5 & $\mathrm{~L}$ & I & 117 & Body & 6 & $19 \mathrm{G}, 22 \mathrm{G}$ \\
\hline 6 & $\mathrm{~L}$ & I & 40 & Tail & 5 & $22 \mathrm{G}, 25 \mathrm{G}$ \\
\hline \multicolumn{7}{|l|}{ Downgraded } \\
\hline 7 & I & $\mathrm{L}$ & 10 & Tail & 3 & $19 \mathrm{G}, 22 \mathrm{G}$ \\
\hline 8 & I & $\mathrm{L}$ & 140 & Head & 2 & $19 \mathrm{G}$ \\
\hline 9 & I & $\mathrm{L}$ & 20 & Tail & 8 & $19 \mathrm{G}, 22 \mathrm{G}$ \\
\hline 10 & I & $\mathrm{L}$ & 17 & Tail & 4 & $25 \mathrm{G}$ \\
\hline
\end{tabular}

EUS-FNA, endoscopic ultrasound with fine-needle aspiration; H, high; I, intermediate; L, low. 
between tumors with concordant versus discordant Ki67 grading.

\section{Concordance between Ki67 grading and mitotic rate grading in surgical resection specimens}

Thirty out of 37 cases (81.1\%) had concordance between Ki67 grading and mitotic rate grading in surgical resected specimens. Concordance was the highest in (22/24) low-grade tumors and (5/5) high-grade tumors, with positive predictive values of $81.5 \%$ and $83.3 \%$ respectively. Only 3 out of 8 intermediate-grade tumors were concordant (Table 3 ).

\section{Prognostic factors for disease-related death and dis- ease recurrence}

Three patients had disease-related death during the follow-up period. All three patients had high-grade Ki67 on both EUS-FNA cytology and surgical resection specimen histology. One had synchronous metastasis during diagnosis with time to disease-related death at 46 months; the other two had disease recurrence within 6 months of surgical resection and had disease-related death at 8 months and 18 months, respectively.

Because time to disease-related death can be long, we defined "poor prognosis" as either disease-related death or disease recurrence and investigated factors leading to those outcomes. ROC curve analysis showed that tumor size $\leq 16.5 \mathrm{~mm}$ would be able to exclude all NF-PNETs with poor prognosis, while tumor size $\leq 22.5 \mathrm{~mm}$ would be able to exclude $87.5 \%$ of NF-PNETs with poor prognosis, area under the curve $=0.743$. Univariate analysis confirmed EUS-FNA Ki67 $\geq 20 \%$ ( $p=0.028$ ), surgical Ki67 $\geq 20 \%$ ( $p=0.028)$, and mitotic rate $>20$ per $10 \mathrm{HPF}$ ( $p=0.005$ ) to be significantly associated with poor prognosis (Table 5).

Table 5. Association between Clinical Features and Poor Prognosis $(n=35)$

\begin{tabular}{lrcc}
\hline & $\boldsymbol{n}$ & Odds ratio & $\boldsymbol{p}$-value \\
\hline Surgical pathology Ki67 $>3 \%$ & 12 & 2.5 & 0.397 \\
Surgical pathology Ki67 $\geq 20 \%$ & 4 & 16.2 & 0.028 \\
EUS-FNA Ki67 $>3 \%$ & 11 & 3 & 0.214 \\
EUS-FNA Ki67 $\geq 20 \%$ & 4 & 16.2 & 0.028 \\
Mitotic rate $>2 / 10 \mathrm{HPF}$ & 9 & 4.6 & 0.086 \\
Mitotic rate $>20 / 10 \mathrm{HPF}$ & 5 & 27 & 0.005 \\
Size $>16.5 \mathrm{~mm}$ & 29 & 48.7 & 0.309 \\
Size $>22.5$ mm & 21 & 7 & 0.104 \\
Positive lymph nodes at time of & 11 & 3 & 0.214 \\
$\quad$ surgery & & & \\
Lymphovascular invasion & 16 & 5.4 & 0.103 \\
\hline
\end{tabular}

EUS-FNA, endoscopic ultrasound with fine-needle aspiration; HPF, high power field.
Because only high-grade Ki67 NF-PNETs were significantly predictive of poor prognosis, our Kaplan Meier analysis investigated time to disease recurrence for high-grade versus lowand intermediate-grade tumors based on surgical Ki67 grading. The Kaplan Meier estimator demonstrated a significantly shorter amount of time to disease recurrence for high-grade tumors compared to low- and intermediate-grade tumors (Fig. 1).

Those with lymphovascular invasion during surgical resection were more likely to have poor prognosis $(37.5 \%)$ compared to those without lymphovascular invasion (10\%). However, this association did not meet statistical significance ( $p=0.103$ ). Similarly, tumor size $>22.5 \mathrm{~mm}$ tended to correlate with poor prognosis (33.3\%) compared to those with smaller tumor size $\leq 22.5 \mathrm{~mm}(6.7 \%)$, but this association was not statistically significant $(p=0.104)$. Positive lymph nodes were not associated with poor prognosis.

\section{DISCUSSION}

\section{Concordance}

This retrospective study is the largest single-center series $(n=37)$ investigating Ki67 concordance between EUS-FNA cytology and surgical pathology specimens in NF-PNETs. Prior series have shown Ki67 concordance rates ranging between $69.2 \%$ and $92 \%$ in all PNETs. ${ }^{8,28-34}$ However, only Larghi

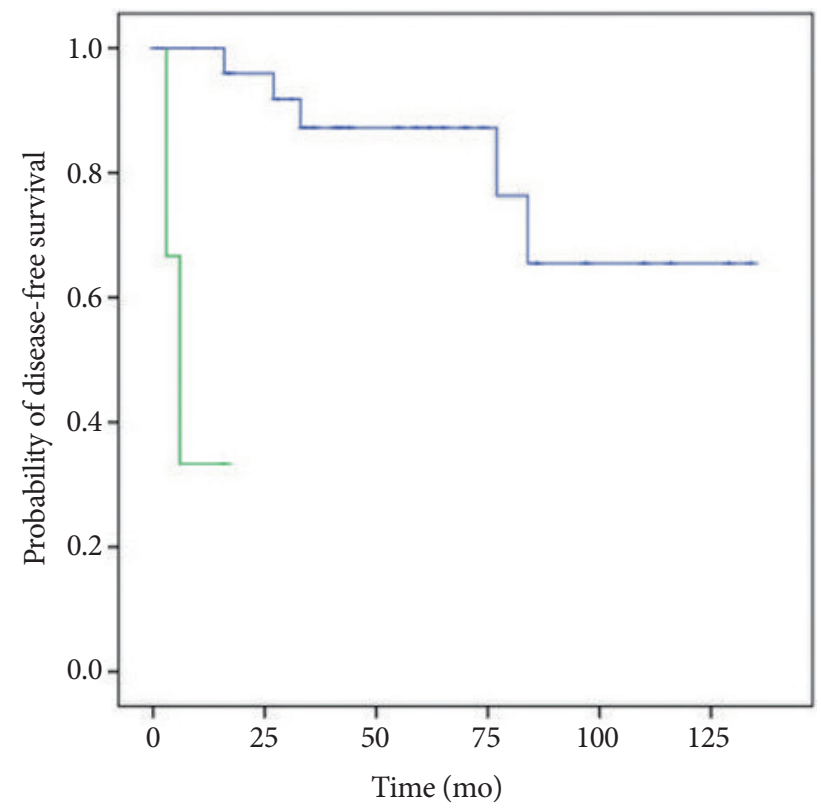

Fig. 1. Time to disease recurrence for low- and intermediate-grade versus high-grade nonfunctioning pancreatic neuroendocrine tumors (NF-PNETs), based on surgical pathology Ki67 of 35 NF-PNET cases.

Green line $=$ NF-PNETs with high-grade Ki67, Blue line $=$ NF-PNETs with lowand intermediate-grade Ki67. 
et al. have validated EUS-FNA as an accurate technique for Ki67 determination in NF-PNETs specifically. ${ }^{29}$ Our study reports a 73\% concordance of Ki67 between cytological and resected tissue samples. Concordance rates were the highest in low- and high-grade PNETs based on surgical Ki67. However, similar to previous studies, intermediate-grade tumors had a lower concordance rate between Ki67 grading on EUS-FNA cytology and surgical resection histology. ${ }^{8,25,27}$ The lower Ki67 concordance rate in our intermediate-grade NF-PNETs could be due to several causes including the following: (1) intratumoral heterogeneity that is reported to be higher in intermediate-grade surgical Ki67 tumors than in low-grade surgical Ki67 tumors; ${ }^{25}$ (2) increased tumor size, which may contribute to increased intratumoral heterogeneity; ${ }^{27}$ or (3) lower cell count from EUS-FNA sampling, as Hasegawa et al. noted increased Ki67 concordance rates from $77.8 \%$ to $90 \%$ when patients with $<2,000$ tumor cells were excluded. ${ }^{8}$ Overall, our Ki67 concordance rate was consistent with prior studies considering both functioning and NF-PNETs, but on the lower end of the range of previously reported concordance rates. This finding may be attributed to increased intratumoral heterogeneity related to larger tumor size, as our average tumor size was $40 \mathrm{~mm}$. Another possibility is that NF-PNETs may have higher intratumoral heterogeneity compared to functioning PNETs, thus contributing to lower Ki67 concordance rates.

\section{Operative versus observational management}

Due to increased use of cross-sectional imaging, incidental detection of NF-PNETs has increased. Risk stratification of these asymptomatic but clinically unpredictable tumors is important in determining management. Many studies have reported elevated Ki67 as a poor prognostic marker for PNETs. Hamilton et al. found that tumors with surgical Ki67 >9\% were more likely to have disease recurrence and decreased overall survival, while Bettini et al. reported surgical Ki67 >5\% to be associated with disease-related mortality. ${ }^{20,22}$ Our study confirms elevated Ki67 as a poor prognostic factor for disease recurrence and disease-related death in both EUS-FNA and surgical samples. Despite lower concordance rates for intermediate-grade NF-PNETs, overall concordance was strong enough that EUS-FNA and surgical Ki67 $\geq 20 \%$ were both associated with disease-related death, with time to disease-related death ranging from 8 months to 46 months despite surgical resection. Additionally, because intermediate-grade NFPNETs demonstrated lower Ki67 concordance and are at risk of being under-graded, NF-PNETs with intermediate-grade Ki67 on EUS-FNA should strongly be considered for surgical resection.

Our data shows that tumor size $\leq 16.5 \mathrm{~mm}$ and $\leq 22.5 \mathrm{~mm}$ would be able to exclude $100 \%$ and $87.5 \%$ of NF-PNETs with poor prognosis, respectively. While increased tumor size had a greater odds ratio of poor prognosis, this association was not statistically significant; thus, we do not recommend using tumor size $>16.5 \mathrm{~mm}$ to define tumors that warrant surgical resection. Based on our data, all NF-PNETs whose EUS-FNA Ki67 grading was upgraded after surgical resection were $\geq 25$ $\mathrm{mm}$. This parallels the Fujimori et al. study where tumors $\geq 20$ $\mathrm{mm}$ had lower rates of concordance between Ki67 obtained from EUS-FNA versus surgical pathology specimen. ${ }^{27}$ These findings strongly support the growing clinical practice and the ENETS's guidelines of observing NF-PNETs with low-grade Ki67 on EUS-FNA up to $20 \mathrm{~mm}^{18}$ Furthermore, our data suggest that a higher threshold of $25 \mathrm{~mm}$ for surgery may be acceptable for low-grade EUS-FNA tumors, as using this cutoff would have excluded any NF-PNETs that should have been resected.

\section{Post-operative surveillance}

Time to disease recurrence was notably shorter for patients with high-grade surgical Ki67 (3 and 6 months) than for lowand intermediate-grade surgical Ki67 (all $>12$ months). Of note, approximately $50 \%$ of our patients had undergone surgical resection within the last five years; therefore, our results may not capture all cases that will develop disease recurrence and disease-related death. We suggest that patients with NFPNETs with surgical Ki67 $\geq 20 \%$ should be monitored at closer intervals post-operatively, while those with low- and intermediate-grade surgical Ki67 may need more prolonged post-operative monitoring for disease recurrence.

In addition to Ki67, our study demonstrated mitotic rate as a separate risk factor for disease recurrence and disease-related death. Mitotic rate is a separate measure used by the ENETS and WHO to grade PNETs. Thus far, studies investigating concordance between Ki67 index and mitotic rate grading have produced conflicting results. ${ }^{828-34}$ We demonstrate $81.1 \%$ concordance rate between Ki67 and mitotic rate grading in surgical resection specimens. Additionally, mitotic rate grading paralleled Ki67 grading in predicting poor prognosis, as tumors with mitotic rate $>20$ per $10 \mathrm{HPF}$ were associated with both disease-related death and disease recurrence. Given the high concordance between surgical Ki67 and mitotic rate as well as the independent risk factor of high-grade mitotic rate for disease recurrence and disease-related death, we support using mitotic rate adjunctively with surgical Ki67 for post-operative surveillance.

Lastly, we found that tumors with lymphovascular invasion during surgical resection had a poor prognosis. This trend was not statistically significant, likely due to small sample size and short duration of follow-up. However, given this finding, pa- 
tients with NF-PNETs with lymphovascular invasion during surgery may benefit from both shorter interval and prolonged course of post-operative surveillance.

\section{Strengths and weaknesses}

The strengths of this study include its size, uniformity, and correlation with natural history. As the nature of the study was retrospective, and all the data were compiled at one medical center, we had access to relevant imaging, EUS-FNA findings, surgical pathology results, as well as a majority of follow-up data. The drawbacks of this study include the relatively short follow-up for disease recurrence and disease-related death, due largely in part to the prolonged disease process. Given that previous studies used different Ki67 and tumor size cutoffs to predict poor prognosis, future studies should investigate whether different Ki67 and tumor size cutoffs would better risk stratify NF-PNETs. Studies with longer follow-up may elucidate the association of low- and intermediate-grade tumors with time to disease recurrence and disease-related death. Finally, investigation of factors leading to discordance of Ki67 between EUS-FNA and surgical pathology (i.e., larger tumor size and increased heterogeneity) can improve our understanding of obtaining optimal tumor samples.

In summary, our study demonstrates that Ki67 indices obtained by EUS-FNA can be used reliably to predict surgical Ki67 indices in NF-PNETs. Our results show that (1) NFPNETs with high-grade Ki67 on EUS-FNA had poor prognosis despite surgical resection; (2) NF-PNETs with intermediate-grade Ki67 on EUS-FNA should be strongly considered for surgical resection; (3) NF-PNETs with low-grade Ki67 on EUS-FNA can be safely observed up to tumor size $20 \mathrm{~mm}$. Post-operatively, patients with low- and intermediate-grade surgical Ki67 tumors may benefit from prolonged surveillance. Mitotic rate and presence of lymphovascular invasion during resection should be considered in post-operative surveillance.

\section{Conflicts of Interest}

The authors have no financial conflicts of interest.

\section{REFERENCES}

1. Halfdanarson TR, Rubin J, Farnell MB, Grant CS, Petersen GM. Pancreatic endocrine neoplasms: epidemiology and prognosis of pancreatic endocrine tumors. Endocr Relat Cancer 2008;15:409-427.

2. Metz DC, Jensen RT. Gastrointestinal neuroendocrine tumors: pancreatic endocrine tumors. Gastroenterology 2008;135:1469-1492.

3. Grimelius L, Hultquist GT, Stenkvist B. Cytological differentiation of asymptomatic pancreatic islet cell tumours in autopsy material. Virchows Arch A Pathol Anat Histol 1975;365:275-288.

4. Kimura W, Kuroda A, Morioka Y. Clinical pathology of endocrine tu- mors of the pancreas. Analysis of autopsy cases. Dig Dis Sci 1991;36:933942.

5. Halfdanarson TR, Rabe KG, Rubin J, Petersen GM. Pancreatic neuroendocrine tumors (PNETs): incidence, prognosis and recent trend toward improved survival. Ann Oncol 2008;19:1727-1733.

6. Fitzgerald TL, Hickner ZJ, Schmitz M, Kort EJ. Changing incidence of pancreatic neoplasms: a 16-year review of statewide tumor registry. Pancreas 2008;37:134-138

7. Kuo EJ, Salem RR. Population-level analysis of pancreatic neuroendocrine tumors $2 \mathrm{~cm}$ or less in size. Ann Surg Oncol 2013;20:2815-2821.

8. Hasegawa T, Yamao K, Hijioka S, et al. Evaluation of Ki-67 index in EUS-FNA specimens for the assessment of malignancy risk in pancreatic neuroendocrine tumors. Endoscopy 2014;46:32-38.

9. Gullo L, Migliori M, Falconi M, et al. Nonfunctioning pancreatic endocrine tumors: a multicenter clinical study. Am J Gastroenterol 2003;98:2435-2439

10. Hill JS, McPhee JT, McDade TP, et al. Pancreatic neuroendocrine tumors: the impact of surgical resection on survival. Cancer 2009;115:741751.

11. Franko J, Feng W, Yip L, Genovese E, Moser AJ. Non-functional neuroendocrine carcinoma of the pancreas: incidence, tumor biology, and outcomes in 2,158 patients. J Gastrointest Surg 2010;14:541-548.

12. Bettini R, Partelli S, Boninsegna L, et al. Tumor size correlates with malignancy in nonfunctioning pancreatic endocrine tumor. Surgery 2011;150:75-82.

13. Lee LC, Grant CS, Salomao DR, et al. Small, nonfunctioning, asymptomatic pancreatic neuroendocrine tumors (PNETs): role for nonoperative management. Surgery 2012;152:965-974.

14. Kishi Y, Shimada K, Nara S, Esaki M, Hiraoka N, Kosuge T. Basing treatment strategy for non-functional pancreatic neuroendocrine tumors on tumor size. Ann Surg Oncol 2014;21:2882-2888.

15. Zhang IY, Zhao J, Fernandez-Del Castillo C, et al. Operative versus nonoperative management of nonfunctioning pancreatic neuroendocrine tumors. J Gastrointest Surg 2016;20:277-283.

16. Regenet N, Carrere N, Boulanger G, et al. Is the 2-cm size cutoff relevant for small nonfunctioning pancreatic neuroendocrine tumors: a French multicenter study. Surgery 2016;159:901-907.

17. National Comprehensive Cancer Network. NCCN clinical practice guidelines in oncology neuroendocrine and adrenal tumors (United States) [Internet]. Plymouth Meeting (PA): NCCN; c2019 [updated 2019 Mar 5; cited 2019 Jul 7]. Available from: https://www.nccn.org/professionals/physician_gls/pdf/neuroendocrine.pdf.

18. Falconi M, Eriksson B, Kaltsas G, et al. ENETS consensus guidelines update for the management of patients with functional pancreatic neuroendocrine tumors and non-functional pancreatic neuroendocrine tumors. Neuroendocrinology 2016;103:153-171.

19. La Rosa S, Sessa F, Capella C, et al. Prognostic criteria in nonfunctioning pancreatic endocrine tumours. Virchows Arch 1996;429:323-333.

20. Bettini R, Boninsegna L, Mantovani W, et al. Prognostic factors at diagnosis and value of WHO classification in a mono-institutional series of 180 non-functioning pancreatic endocrine tumours. Ann Oncol 2008:19:903-908

21. Alexiev BA, Darwin PE, Goloubeva O, Ioffe OB. Proliferative rate in endoscopic ultrasound fine-needle aspiration of pancreatic endocrine tumors: correlation with clinical behavior. Cancer 2009;117:40-45.

22. Hamilton NA, Liu TC, Cavatiao A, et al. Ki-67 predicts disease recurrence and poor prognosis in pancreatic neuroendocrine neoplasms. Surgery 2012;152:107-113.

23. Unno J, Kanno A, Masamune A, et al. The usefulness of endoscopic ultrasound-guided fine-needle aspiration for the diagnosis of pancreatic neuroendocrine tumors based on the World Health Organization classification. Scand J Gastroenterol 2014;49:1367-1374.

24. Cherenfant J, Talamonti MS, Hall CR, et al. Comparison of tumor markers for predicting outcomes after resection of nonfunctioning pancreatic neuroendocrine tumors. Surgery 2014;156:1504-1510; discussion 
1510-1511

25. Díaz Del Arco C, Esteban López-Jamar JM, Ortega Medina L, Díaz Pérez JÁ, Fernández Aceñero MJ. Fine-needle aspiration biopsy of pancreatic neuroendocrine tumors: correlation between Ki-67 index in cytological samples and clinical behavior. Diagn Cytopathol 2017;45:2935.

26. McLean AM, Fairclough PD. Endoscopic ultrasound in the localisation of pancreatic islet cell tumours. Best Pract Res Clin Endocrinol Metab 2005;19:177-193.

27. Fujimori N, Osoegawa T, Lee L, et al. Efficacy of endoscopic ultrasonography and endoscopic ultrasonography-guided fine-needle aspiration for the diagnosis and grading of pancreatic neuroendocrine tumors. Scand J Gastroenterol 2016;51:245-252.

28. Piani C, Franchi GM, Cappelletti C, et al. Cytological Ki-67 in pancreatic endocrine tumours: an opportunity for pre-operative grading. Endocr Relat Cancer 2008;15:175-181.

29. Larghi A, Capurso G, Carnuccio A, et al. Ki-67 grading of nonfunctioning pancreatic neuroendocrine tumors on histologic samples obtained by EUS-guided fine-needle tissue acquisition: a prospective study. Gastrointest Endosc 2012;76:570-577.
30. Carlinfante G, Baccarini P, Berretti D, et al. Ki-67 cytological index can distinguish well-differentiated from poorly differentiated pancreatic neuroendocrine tumors: a comparative cytohistological study of 53 cases. Virchows Arch 2014;465:49-55.

31. Farrell JM, Pang JC, Kim GE, Tabatabai ZL. Pancreatic neuroendocrine tumors: accurate grading with Ki-67 index on fine-needle aspiration specimens using the WHO 2010/ENETS criteria. Cancer Cytopathol 2014;122:770-778.

32. Weynand B, Borbath I, Bernard V, et al. Pancreatic neuroendocrine tumour grading on endoscopic ultrasound-guided fine needle aspiration: high reproducibility and inter-observer agreement of the Ki-67 labelling index. Cytopathology 2014;25:389-395.

33. Rebours V, Cordova J, Couvelard A, et al. Can pancreatic neuroendocrine tumour biopsy accurately determine pathological characteristics? Dig Liver Dis 2015;47:973-977.

34. Boutsen L, Jouret-Mourin A, Borbath I, van Maanen A, Weynand B. Accuracy of pancreatic neuroendocrine tumour grading by endoscopic ultrasound-guided fine needle aspiration: analysis of a large cohort and perspectives for improvement. Neuroendocrinology 2018;106:158-166. 